\title{
A newly identified mutation in an IPF1 binding site of the insulin gene promoter may predispose to type 2 diabetes mellitus
}

\author{
M. T. Malecki • P. Lebrun • M. Pezzolesi • \\ J. H. Warram • A. S. Krolewski • U. S. Jhala
}

Received: 26 January 2006 / Accepted: 19 April 2006 / Published online: 2 June 2006

(C) Springer-Verlag 2006

To the Editor: Autosomal dominant diabetes is a heterogeneous subtype of the disease usually characterised by early onset and a primary defect in insulin secretion. To date, six genes have been found to be associated with early-onset autosomal dominant diabetes, also called MODY. Five of them are transcription factors directly or indirectly involved in the process of insulin gene expression [1]. Many regulatory enhancer elements, including the critical glucose response elements, have been identified in the insulin promoter, several within the proximal $300-400$ bp region. Remarkably, most transcription factors known to be associated with MODY have been shown to regulate insulin gene expression directly through these sites [2]. A search for mutations in the most proximal part of the insulin promoter in a large set of pedigrees with autosomal dominant diabetes

M.T. Malecki and P. Lebrun contributed equally to this work.

M. T. Malecki • M. Pezzolesi · J. H. Warram • A. S. Krolewski Section on Genetics and Epidemiology, Joslin Diabetes Center, Harvard Medical School,

Boston, MA, USA

P. Lebrun

INSERM U145, Faculty of Medicine, IFR50,

Nice, France

\section{U. S. Jhala $(\square)$}

Islet Research Labs, The Whittier Institute,

University of California San Diego,

9894 Genesee Avenue \#225,

La Jolla, CA 92037, USA

e-mail: ujhala@ucsd.edu

\section{T. Malecki}

Department of Metabolic Diseases, Jagellonian University, Medical College,

Krakow, Poland resulted in the identification of a $-80 \mathrm{~A} \rightarrow \mathrm{G}$ point mutation within the insulin promoter factor 1 , homeodomain transcription factor (IPF1, also known as MODY4 and PDX1) binding site of the glucose response region. The role of this mutation in susceptibility to diabetes is discussed below.

To test the hypothesis that alterations in the proximal regulatory regions of the insulin gene occur in a subset of patients from families with autosomal dominant diabetes, we examined $323 \mathrm{bp}$ immediately upstream of the transcription start site by direct sequencing using the following primers: forward 5'-tcctggtctaatgtggaaagtggec-3'; reverse: 5'-atggcagaaggacagtgatctg- 3 '. We screened DNA samples from 94 index cases in families of European descent confirmed to have diabetes segregating as an autosomal dominant disorder. The ascertainment and characteristics of these families have been described in a previous study [3]. Of the 94 families, 45 families had early-onset diabetes meeting MODY criteria, while in 49 families the disease was diagnosed later in life and was considered autosomal dominant late-onset type 2 diabetes. The Committee on Human Subjects of the Joslin Diabetes Center approved the study protocol; informed consent procedures and written consent to participate were obtained.

Sequence analysis of the insulin gene promoter in these individuals revealed substitution of an $A$ to $G$ at position -80 in one subject. The mutation was confirmed by cloning the PCR product into a TA cloning vector (pCR 2.1; Invitrogen, Carlsbad, CA, USA) and sequencing clones representing each allele. The mutation changes an evolutionarily well-conserved adenine base residue within the A1 box that constitutes an IPF1 binding site [4]. The A1 box is part of a so-called 'mini-enhancer' that is sufficient to confer glucose responsiveness to the insulin gene. The 


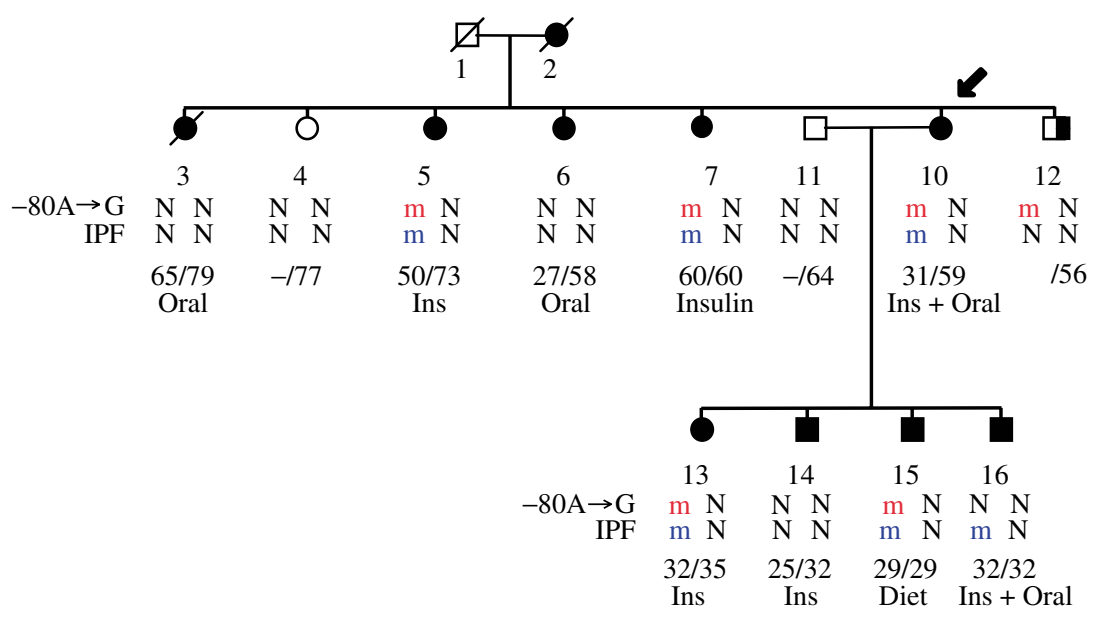

Fig. 1 Pedigree, genotypes and clinical characteristics of Family A with data for the examined individuals, founders and deceased diabetic patients. The segregation of $-80 \mathrm{~A} \rightarrow \mathrm{G}$ and $\mathrm{D} 76 \mathrm{~N}$ mutations is shown Closed symbols, subject with diabetes; half closed symbols, subjects with IGT; open symbols, subjects with NGT. The first line under the symbols is the identification number. The second and third lines are

$-80 \mathrm{~A} \rightarrow \mathrm{G}$ mutation was not present in a set of 96 unrelated control individuals, all Caucasians of European origin.

Interestingly, this variant was identified in family A, where an IPF1 gene D76N mutation was previously found (M. T. Malecki, A. S. Krolewski, unpublished data) (Fig. 1). The D76N mutation has been linked in some $[5,6]$, but not the genotype at insulin promoter nucleotide -80 of insulin promoter and at residue 76 of the IPF gene, respectively, for examined individuals: $N$, normal allele; $m$, mutant allele. The fourth line is the age at diabetes diagnosis for affected members, age at death for deceased members, and age at examination for others. The last line is treatment for diabetes at the time of examination. An arrow indicates

all studies [7], with susceptibility to type 2 diabetes. The index case from this family (individual 10) was screened for mutations in all remaining MODY genes. This search, however, produced negative results. DNA from 12 family members was tested; nine of whom had diabetes, while one had IGT. Of these ten affected individuals, six were carriers a

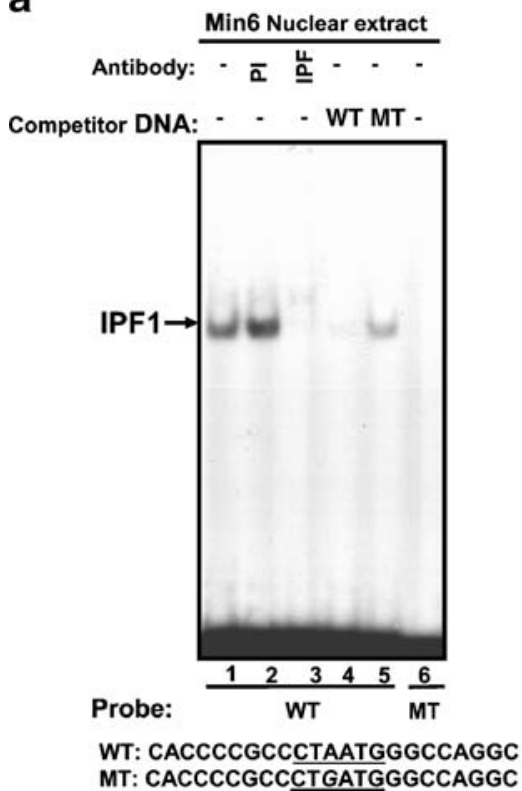

Fig. 2 Functional characterisation of the $-80 \mathrm{~A} \rightarrow \mathrm{G}$ mutation. a Electro mobility shift assay was performed using nuclear extracts from Min6 insulinoma cells, and $\gamma-\mathrm{P}^{32}$ ATP labelled wild-type (WT) (lanes 1-5) and mutant (MT) DNA (lane 6) corresponding to a short stretch (-91$70 \mathrm{bp}$ ) around the $\mathrm{A} \rightarrow \mathrm{G}$ mutation as described [8]. Specificity of DNA binding was ascertained using 50-fold excess unlabelled wildtype (lane 4) or mutant DNA (lane 5), and identification of IPF1 b

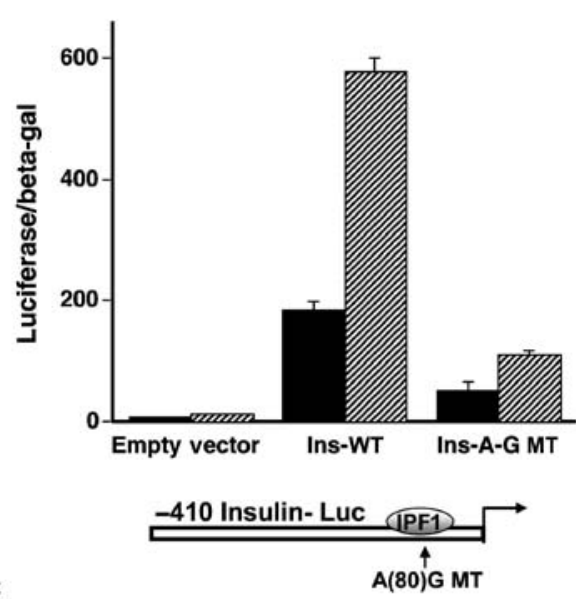

binding activity was determined using an anti-IPF1 antibody (lane 3). Pre-immune serum was used as a control (lane 2). b Wild-type and $-80 \mathrm{~A} \rightarrow \mathrm{G}$ mutant human insulin promoter-luciferase reporter constructs were transfected into Min6 insulinoma cells ( -410 to $+10 \mathrm{bp})$ and treated with low ( $3 \mathrm{mmol} / \mathrm{l}$, solid bars) or high $(24 \mathrm{mmol} / \mathrm{l}$, hatched bars) glucose. Beta-galactosidase ( $\mathrm{gal}$ ) was used as a transfection control and to normalise luciferase activity 
of the $-80 \mathrm{~A} \rightarrow \mathrm{G}$ substitution in the insulin promoter, five were diabetic patients and one had IGT. All five diabetic patients with the $-80 \mathrm{~A} \rightarrow \mathrm{G}$ mutation also carried an IPF1 D $76 \mathrm{~N}$ mutation. Additionally, we identified one patient with the $\mathrm{D} 76 \mathrm{~N}$ mutation but without the rare promoter variant. There were also three individuals who had diabetes but were not carriers of insulin promoter and IPFI mutations.

Clearly, the segregation of diabetes and of both mutations in this family did not provide an unequivocal answer with regard to the role of this newly identified promoter variant in susceptibility to the disease. Interestingly, the functional data provided evidence for a potential biological role of the $-80 \mathrm{~A} \rightarrow \mathrm{G}$ mutation. First, we examined its impact on IPF1 binding to the mutant promoter using electro mobility shift assay (EMSA). As shown in Fig. 2a, the wild-type, $\gamma$-p32ATP radiolabelled probe showed robust binding to protein/s from beta cell nuclear extracts confirmed as IPF1 using specific antibodies (lanes 1-3). By contrast, and as seen in lane 6, the $-80 \mathrm{~A} \rightarrow \mathrm{G}$ mutant probe failed to bind IPF1. This mutation lies in the IPF1 binding site of a composite enhancer element, which synergistically mediates glucose-dependent activation of the insulin promoter with other transcription factors, including NeuroD1 and MafA [4]. Such a failure of IPF1 to bind DNA had a strong (five-fold) effect on the capacity of the mutant promoter to drive both basal and glucose-induced luciferase reporter gene expression (Fig. 2b).

In summary, sequence differences in the proximal part of the insulin gene promoter are not a major cause of MODY or autosomal dominant late-onset type 2 diabetes. This is the first report to identify a naturally occurring mutation in a key enhancer element of the insulin gene promoter. All the carriers of this mutation were affected, but the genetic background of diabetes in this family seemed to be complex and certainly not limited to the $-80 \mathrm{~A} \rightarrow \mathrm{G}$ substitution. While the results of functional studies showed that the mutation had a strong impact on activity of the insulin promoter, most carriers of the newly identified variant were characterised by a rather modest diabetic phenotype. This discrepancy could be explained by the presence of up to three additional IPF1 binding sites on each allele and the full complement of IPF1 binding sites on the other allele. It is possible that impaired function of the insulin promoter with the $-80 \mathrm{~A} \rightarrow \mathrm{G}$ substitution could be unmasked in humans in the presence of other genetic and environmental factors such as obesity and insulin resistance. Thus, we postulate that $-80 \mathrm{~A} \rightarrow \mathrm{G}$ mutation in the insulin gene promoter may contribute to the genetic background of type 2 diabetes mellitus as a part of an oligogenic or polygenic inheritance.

Acknowledgements $\quad$ M. T. Malecki is supported by the NIH FIRCA 1 R03 TW01351-01 Grant. A. S. Krolewski was supported by NIH DK47475 Grant. U. S. Jhala was funded by the Hillblom Foundation.

\section{References}

1. Fajans SS, Bell GI, Polonsky KS (2001) Molecular mechanisms and clinical pathophysiology of maturity-onset diabetes of the young. N Engl J Med 345:971-980

2. Melloul D, Marshak S, Cerasi E (2002) Regulation of insulin gene transcription. Diabetologia 45:309-326

3. Malecki MT, Jhala US, Antonellis A et al (1999) Mutations in NEUROD1 gene are associated with the development of type 2 diabetes mellitus. Nat Genet 23:323-328

4. German M, Ashcroft S, Docherty K et al (1995) The insulin gene promoter. A simplified nomenclature. Diabetes 44:1002-1004

5. Macfarlane WM, Frayling TM, Ellard S et al (1999) Missense mutations in the insulin promoter factor-1 gene predispose to type 2 diabetes. J Clin Invest 104:R33-R39

6. Hani EH, Stoffers DA, Chevre JC et al (1999) Defective mutations in the insulin promoter factor-1 (IPF-1) gene in late-onset type 2 diabetes mellitus. J Clin Invest 104:R41-R48

7. Elbein SC, Karim MA (2004) Does the aspartic acid to asparagine substitution at position 76 in the pancreas duodenum homeobox gene $(P D X 1)$ cause late-onset type 2 diabetes? Diabetes Care 27:1968-1973

8. Sharma S, Jhala US, Johnson T et al (1997) Hormonal regulation of an islet-specific enhancer in the pancreatic homeobox gene STF-1. Mol Cell Biol 17:2598-2604 